\title{
Cell-based APPRoAches in DRUg DEVElopment A CONCISE REVIEW
}

\author{
Ievgeniia Kocherova ${ }^{1}$, Bartosz Kempisty ${ }^{1,2,3,4}$, Greg Hutchings ${ }^{1,5}$, Lisa Moncrieff ${ }^{2,5}$, Claudia Dompe ${ }^{2,5}$, \\ Krzysztof Janowicz ${ }^{1,5}$, Jim Petitte ${ }^{6}$, Jamil A. Shibli ${ }^{7}$, Paul Mozdziak ${ }^{8}$
}

\begin{abstract}
In vitro models represent an alternative technique to in vivo or ex vivo studies in the drug development process. Cell-based assays are used to measure the level of proliferation and toxicity, as well as activation of signalling pathways and changes in morphology in cultivated cells. The studies conducted in vitro are aimed to estimate the newly synthesised drugs' ability to permeate biological barriers and exert their therapeutic or cytotoxic effects. However, more than half of all studied drugs fail in the second or third phase of clinical trials due to a lack of confirmed efficacy. About a third of drugs fail because of safety issues, such as unacceptable levels of toxicity. To reduce attrition level in drug development, it is crucial to consider the implementation of translational phenotypic assays as well as to decipher various molecular mechanisms of action for new molecular entities. In this review, we summarise the existing cell-based methods most frequently used in the studies on drugs, taking into account their advantages and drawbacks.
\end{abstract}

Running title: Cell-based approaches in drug development

Keywords: drug development, cell-based model, drug screening, cytotoxicity

\footnotetext{
${ }^{1}$ Department of Anatomy, Poznań University of Medical Sciences, Poznań, Poland

${ }^{2}$ Department of Histology and Embryology, Poznań University of Medical Sciences, Poznań, Poland

${ }^{3}$ Department of Obstetrics and Gynaecology, University Hospital and Masaryk University, Brno, Czech Republic

${ }^{4}$ Department of Veterianary Surgery, Institute of Veterinary Medicine, Nicolaus Copernicus University in Toruń, Toruń, Poland

${ }^{5}$ The School of Medicine, Medical Sciences and Nutrition, University of Aberdeen, Aberdeen, UK

${ }^{6}$ Prestage Department of Poultry Science, North Carolina State University, Raleigh, USA

${ }^{7}$ Department of Periodontology and Oral Implantology, Dental Research Division, University of Guarulhos, Guarulhos, Brazil

${ }^{8}$ Physiology Graduate Program, North Carolina State University, Raleigh, USA

* Correspondence: bkempisty@ump.edu.pl

Full list of author information is available at the end of article
} 


\section{Introduction}

Drug development represents a long and expensive process. It involves several stages from target identification to lead discovery and optimisation of the final drug composition, preclinical and clinical trials culminating in approval for clinical use.

Cell-based studies represent an important stage of the drug discovery process. In vitro cell models provide a relatively simple, reproducible and cost-effective tool to avoid large-scale animal testing. Properly selected cell models and culture conditions allow one to answer the fundamental questions of pharmacological and biochemical nature and are necessary to assess drug safety, efficacy and the mechanism of action. For this purpose, investigated compounds are added to the culture medium and then their effects at the cellular and molecular level might be analysed. Additionally, the use of cell cultures in toxicological assays requires small amounts of tested substances, reducing the cost related to expensive and time-consuming in vivo studies [1].

Moreover, tests using human cells may be more relevant than in vivo animal tests [2]. The main advantage of in vivo model is the integration of dynamic components of the blood circulation, different tissue layers and the other factors that can influence drug absorption and effectiveness on the systemic level. However, the essential drawback of in vivo models is that it is impossible to separate the variables involved in the process of absorption and identify the individual rate-limiting factors [3].

Currently, more than half of all studied drugs fail in the second or third phase of clinical trials due to a lack of confirmed efficacy. About third of drugs fail because of safety issues, such as unacceptable toxicity [4]. As attrition rates in drug development remain high, there is an urgent need for improvement of screening methods allowing for better precision and reliable estimation of drug effects. The present review focuses on the existing cell-based methods most frequently used in the study of drugs, taking into account their advantages and drawbacks.

\section{Cytotoxicity evaluation}

Cytotoxicity is one of the crucial indicators for biological evaluation in vitro studies. Cell viability and cytotoxicity tests are based on the measurement of cell membrane permeability, cell adherence, activity of enzymes and co-enzymes, ATP production and nucleotide uptake level [2]. The cytotoxic effect of the tested compound is characterised by the determination of its concentration for which the proliferation of treated cells is inhibited by $50 \%$ (IC50) when compared to those of control cells [1]. Cytotoxicity studies are performed in order to assess the viability of cells in the presence of a particular drug substance using a great variety of tests. The wide range of cytotoxicity and cell viability assays can be classified among others according to measurement types of endpoints [2]:

- dye exclusion (eosin, Trypan blue, erythrosine $\mathrm{B}$, Congo red assays);

- colourimetric assays (MTT/MTS, XTT, WST1, WST-8, LDH, SRB, NRU and crystal violet assays);

- fluorometric assays (Alamar Blue/resazurin assay, CFDA-AM);

- luminometric assays (ATP assay, real-time viability assay).

Determination of membrane integrity is possible using a dye exclusion assay, which is one of the simplest and widely used methods. Of the dyes listed, Trypan blue has been used the most extensively to assess the percentage of viable cells [5]. After the addition of Trypan blue into cell suspension, viable cells will have a clear cytoplasm, whereas dead cells will have a blue cytoplasm. The number of viable and dead cells per unit volume is determined by light microscopy using hemacytometer [6].

While the staining protocol is quite fast and simple it is difficult to process a large number of samples simultaneously, particularly when the exact timing of progressive cytotoxic effects is taken into consideration. Moreover, Trypan blue staining cannot detect the cells that are alive but losing their metabolic activity. An important disadvantage of Trypan blue is its toxic side effect on mammalian cells [7]. Additionally, such dyes as Trypan blue are not recommended for use on monolayer cell cultures, as they are designed for cell suspensions. This means that monolayer cells must first be trypsinised.

By contrast, colourimetric assays are applicable for both adherent and suspended cell cultures. MTT assay is a widely used colourimetric method to measure cell death. The reduction of yellow tetrazolium dye (MTT) by mitochondrial dehydrogenase leads to a coloured formazan product accumulation - this process represents an underlying chemical reaction in every tetrazolium based assay [8]. Various organic solvents, such as isopropanol / hydrochloride acid solution might be used to dissolve purple formazan crystals. XTT, MTS, WST-1, and WST-8 assays represent the improved versions of MTT [1]. The final product of the reaction is soluble in water or cell culture medium, however the solubility of these dyes is substantially reduced, and the concentrations of 1-2 $\mathrm{mg} / \mathrm{ml}$ should be applied instead of $0.2-0.5 \mathrm{mg} / \mathrm{ml}$ used in the case of the original MTT [8].

LDH (lactate dehydrogenase) cytotoxicity assay is another colourimetric method of determining cellular cytotoxicity. The assay quantitatively measures the stable, cytosolic LDH, which releases from damaged cells. LDH is an enzyme which is normally located within the cell cytoplasm. Reduced cell viability is associated with increased leakiness of the plasma membrane, allowing for LDH release into the 
cell culture medium. The released LDH can be measured with a coupled enzymatic reaction resulting in the conversion of a tetrazolium salt (iodonitrotetrazolium) into a red colour formazan by catalyst (diaphorase) [9]. The major limitation of this cytotoxicity assay is that serum used for culture medium supplementation and some other compounds possess inherent LDH activity and cause extremely high background readings. For this reason, LDH assay is limited to serum-free or low-serum culture conditions [2].

Among the various methods used for cell viability and cytotoxicity assessment, resazurin-based assay (Alamar Blue) seems to exhibit all features of an optimal, reliable test - it is one-step, sensitive, non-toxic for cells, and cost-effective [10]. In living cells, resazurin is known to intercept the electrons from the mitochondrial electron transport chain. In this way, non-fluorescent resazurin (blue, oxidised) is being converted to its reduced fluorescent form - resorufin (pink), with an absorption maximum at $601 \mathrm{~nm}$. The metabolic activity of cells is proportional to the amount of produced resorufin. Resazurin has been used extensively in cell proliferation, growth, and toxicity studies of cells types ranging from human to bacterial cells [11]. However, the fact that bacteria are able to reduce resazurin indicates the crucial role of conducting clean, contamination-free cell cultures in obtaining reliable results.

\section{Clonogenic cell survival assay}

The clonogenic assay has been initially developed for evaluating the impact of ionising radiation on mammalian cell culture [12]. Nowadays, it is commonly used for studying the effects of experimental drugs on tumour cells. The cells are considered clonogenic if they maintain their reproductive ability to proliferate and form a large colony or a clone [13]. The colony formation assay consists of plating a known number of cells, allowing them to settle and grow for 1-3 weeks, fixing and staining them with crystal violet, and counting the cells that formed colonies. The colony is defined to comprise of at least 50 cells. The colonies consisting of cells with damaged nuclei or that are only formed of a small number of cells should be considered non-viable, as they are not able to undergo "unlimited" divisions. The loss of cells' ability to form the colonies can be related to the antitumor effect of studied agents by a dose-survival curve [14].

\section{DNA synthesis assay}

The inhibition of cell proliferation can reflect the cytotoxic effect of a studied compound and therefore assessing the proliferation activity is useful when testing the effectiveness of new anti-cancer drugs, as well as the safety of various therapeutic agents [12].

During $S$ phase of the cell cycle genome replication occurs, when DNA polymerases incorporate nucleosides into newly synthesised DNA strands. The process of DNA synthesis is relatively specific for cell division and therefore can be considered a marker of proliferation activity of cells [15]. Nucleoside-analog incorporation assays rely on the introduction of chemically or radioactively labelled nucleosides, which subsequently become incorporated into DNA strands synthesised during $\mathrm{S}$ phase. Further analysis allows for the quantitative assessment of proliferation level, based on the amount of labelled nucleoside utilised by cells.

A classic example of the DNA synthesis assay is radioactive tagging with $3 \mathrm{H}$-labeled thymidine $(3 \mathrm{H}$ T). During this incorporation assay, the excess of radiolabeled thymidine is added to cell samples and allowed to incubate for several days. The measurement of the level of incorporated 3H-T is performed using a liquid scintillation counter. This assay is commonly regarded as reliable and accurate. It can be performed in experiments in vitro and ex vivo, but not in vivo. The other disadvantage is that $3 \mathrm{H}-\mathrm{T}$ is an endpoint assay because of the DNA extraction step, and so no further studies can be performed with the treated cells. Moreover, radioactive reagents such as 3H-T must be used and disposed of with caution.

Nowadays, the use of radioactive materials is limited, and another protocol which uses 5-bromo-2'-deoxyuridine (BrdU), also a synthetic thymidine analogue, is usually preferred [12]. Incorporated BrdU is detected by BrdU-specific antibody and can be measured as a colourimetric, chemiluminescent or fluorescent signal. This technique is suitable for immunohistochemistry or immunocytochemistry, in-cell ELISA, flow cytometry and high-throughput screening (HTS). Besides avoiding the use of radioactive substances, the advantage of this method is that it can be used not only in vitro or ex vivo but also in vivo. BrdU can be administrated into an animal via injection or addition to the drinking water. BrdU remains stably incorporated into DNA strands, persisting up to several months. However, similarly to $3 \mathrm{H}-\mathrm{T}$ it is an endpoint assay because the cell membrane permeabilisation and fixation are needed for staining. Both assays cannot identify cells that have undergone numerous divisions $[16,17]$.

\section{Stress reduction assays}

Stress reduction assays are aimed to assess the elimination or reduction of the stresses and toxins that cause cell damage. This approach is widely used for studies on drugs for neurodegenerative diseases. For example, in the case of Alzheimer's disease (AD), the characteristic pathological markers are represented by amyloid plaques consisting of amyloid-beta $(A \beta)$ peptide and neurofibrillary tangles formed by microtubule-associated protein tau (MAPT) [18]. For this reason, phenotypic screens are performed to search for compounds that reduce $A \beta$ or MAPT levels, using an established cell line that overexpresses $A \beta$ or tau protein $[19,20]$. In the case of Parkin- 
son's disease (PD), the loss of dopaminergic neurons in substantia nigra results in inhibited dopaminergic stimulation, leading to motor dysfunction. Therefore, agents improving disease progression must improve the degeneration of dopaminergic neurons under the conditions of induced cytotoxicity in vitro [21].

Stress reduction screenings are also useful for searching for compounds with antioxidative and anti-inflammatory effects. Antioxidative properties can be readily assayed in HTS format, measuring the level of reactive oxygen species (ROS) produced by cells or in chemical assays such as DPPH free radical scavenging method $[22,23]$. However, the chemical assays only provide information about free radical scavenging potential of the studied compounds. In contrast, cell-based approaches allow estimating the balance between the production of ROS in treated cells and the activity of antioxidative cell defence. Additionally, compounds with anti-inflammatory properties can be screened against lipopolysaccharide (LPS)-induced nitric oxide (NO) production, and tumour necrosis factor- $\alpha$ (TNF- $\alpha$ ) and interleukin-1 $\beta$ (IL-1 $\beta$ ) expression [24].

\section{Drug permeability studies}

Drug absorption across biological membranes represents a complex multi-pathway process. Drugs can penetrate cells via different routes $[3,25]$ :

- transcellular route:

- passively through the cell membrane,

- actively through carrier-mediation - active or facilitated diffusion,

- endocytosis,

- paracellular route - via the junction connections between the adjacent cells.

Various efflux transporters incorporated into cell membranes, such as P-glycoprotein (P-gp) can limit drug absorption [26]. Some epithelial enzymes are involved in metabolising drugs to alternate compounds, which may be further absorbed. Finally, receptor-mediated endocytosis also contributes to the process of absorption. Among the several pathways the molecules follow to penetrate cell membranes, diffusion via lipid- and carrier-mediated transport is particularly important regarding the pharmacokinetic mechanisms [27]. The preferred pathway for specific drug absorption or transport depends on its physicochemical characteristics as well as the membrane features. In general, lipophilic drugs tend to cross the biological membrane by the transcellular pathway. In contrast, hydrophilic drugs pass the membrane paracellulary through cell junctions, although hydrophilic drugs can also cross via transporters [3].

Quantitative permeation through the cell monolayer can be defined by the permeation coefficient (Pc) [28]:

$\mathrm{Pc}=\mathrm{V} / \mathrm{A} \times \mathrm{Co} \times \mathrm{dC} / \mathrm{dt}[\mathrm{cm} / \mathrm{s}]$

where:
$\mathrm{V}$ - culture medium volume $\left(\mathrm{cm}^{3}\right)$,

A - surface area of the membrane $\left(\mathrm{cm}^{2}\right)$,

Co - initial concentration of the component, where the studied compound was introduced $\left(\mu \mathrm{g} / \mathrm{cm}^{3}\right)$,

$\mathrm{dC} / \mathrm{dt}$ - increase of concentration after the compound administration.

Permeability data obtained from different cell models can vary between laboratories, even though good in vitro - in vivo correlations with human data have been obtained. Among the main factors affecting the permeability data are the laboratory materials, cell source, and nonstandardized assays. In order to develop suitable permeability procedures several criteria are required, such as confirmed by standardised procedures experimental protocol, with a strong correlation with human data, and where high permeability internal standards, reference compounds, markers for tissue integrity and cell viability have to be used [29]. Among the post-experimental factors, the variability associated with the analytical and statistical method is an important aspect to take into consideration, as well as the estimation method and underlying mathematical assumptions, which represent an aspect often neglected [30].

There are various drug administration site-specific models, such as intestinal, nasal, pulmonary, vaginal, rectal, ocular and skin, that have been established and examined in vitro. Drug administration through the oral route is considered as the preferred route, mainly due to patient's compliance to therapeutics, reduced risk of infection, as well as lack of pain associated with other invasive routes. However, the transport of drug substances via the intestinal membrane is a complex process that includes the passage of compounds across several functional pathways concurrently. Caco-2 cell monolayers have been by far the most frequently used cell model to simulate the intestinal epithelium and predict the flux drugs across small intestinal tissue [31]. One of the drawbacks associated with the use of Caco-2 model is the heterogeneity of the parental cell line leading to the selection of different cell subpopulations becoming prominent in culture [32]. Co-cultures comprising different cell types have been investigated in order to more closely reproduce the histological structure of intestinal epithelium. It has been reported that the Caco-2/HT29/Raji $\mathrm{B}$ triple co-culture was reliable in obtaining a more physiological and reproducible in vitro model of the intestinal barrier, which was suitable to study drug absorption including compounds associated with nanocarriers [3]. Many of the co-culture systems become of great interest in the field of academic research but are still not commonly used in the pharmacological industry.

The blood-brain barrier (BBB) is another example of a complex anatomical and functional structure that must be precisely simulated in vitro to de- 
velop new neuropharmaceuticals. BBB represents a physical restraint in the pharmacological treatment of neurological disorders, as it prevents most of the pharmaceuticals from reaching the neurons. There are different ways to mimic the specific boundary between blood vessels and nerve tissue in vitro. From in vitro co-culture models, generally, the ones that best mimic in vivo $\mathrm{BBB}$ anatomical conditions use brain endothelial cells with astrocytes and/or pericytes [33]. To study BBB model efficiency, several barrier properties should be analysed. One of these commonly assessed properties is paracellular permeability, which is measured through TEER values. These values are usually obtained through a technique based on the use of two electrodes, one placed in the upper (mimics blood) and the other in the lower (mimics brain) chambers, separated by the BBB model cultured on a semipermeable membrane (Transwell). Another method to assess BBB in vitro model is to check for the permeability of specific molecules compared with their permeability values in vivo [34].

In the last decades, most of the current successful $\mathrm{BBB}$ in vitro models are based on brain endothelial cells primary cultures due to their high TEER values and a low cellular passage which closely resemble in vivo BBB properties. However, there is a limitation in the availability of the primary cells. The samples are usually acquired either from biopsies or autopsies, so these tissues often cannot be considered a healthy cell source [35]. For these reasons, primary cells might not be an appropriate choice for every in vitro testing, especially when it requires scalability to be cost-effective. On the other hand, immortalised cell lines retain their viability over numerous passages with a higher experimental reproducibility compared with primary cells.

\section{Two- (2D) and three-dimensional (3D) culture models}

To get reliable results, the in vitro models used as the testing platform should perform similarly to the cells in vivo. The key element of these models - cultured cells - represent the most critical part of such an approach, since the results are obtained based on cellular responses to studied drugs and external stimuli. To date, the majority of cell-based assays implies traditional two-dimensional (2D) monolayer cells culture [36]. Although 2D cultures have proven to be a valuable method for cell-based studies, its limitations have been increasingly recognised, since it does not adequately take into account the natural 3D environment of cells in vivo. The limited physiological relevance of $2 \mathrm{D}$ culture models is determined by the interconnections between the cells and the extracellular matrix (ECM), which is known to significantly affect cellular phenotype and function in a tissue-specific manner [4]. As a result, 2D cell culture tests sometimes reveal misleading data, nonpredictive for in vivo responses
[36]. Application of 3D cell culture in HTS, however, remains a challenge. Aside from the question of labour intensiveness and material cost, scalability, reproducibility, incorporation into an automated screening setup and compatibility with currently available assays are areas of concern [4].

\section{Conclusions}

An ideal cell-based culture model would simulate a tissue-specific physiological or disease-specific environment. Such a model would include cell-to-cell contact and cell-to-ECM interactions, as well as tissue-specific stiffness, oxygen, nutrient and metabolic waste product gradients. Most culture techniques currently do not meet all of the above criteria but rather have their own advantages and drawbacks. Thus, it is important to choose the most appropriate cell-based culture model for a specific application in combination with the proper selection of relevant assays. This approach will allow for the estimation of the most significant parameters in the treated cells and obtaining reliable and physiologically relevant results.

\section{Ethical approval}

The conducted research is not related to either human or animal use.

\section{Acknowledgements}

Not applicable.

\section{Corresponding author}

Bartosz Kempisty PhD, Department of Histology and Embryology, Department of Anatomy, Poznan University of Medical Sciences, 6 Święcickiego St., 60-781 Poznań, Poland Tel./Fax: +48 618546418 / +4861 8546440, e-mail: bkempisty@ump.edu.pl.

\section{Conflict of interest statement}

The authors declare they have no conflicts of interest.

\section{References}

1. Amelian A, Wasilewska K, Megias D, Winnicka K. Application of standard cell cultures and 3D in vitro tissue models as an effective tool in drug design and development. Pharmacol Rep. 2017;69:861-70; DOI:10.1016/j.pharep.2017.03.014.

2. Aslantürk ÖS. In vitro Cytotoxicity and Cell Viability Assays: Principles, Advantages, and Disadvantages. Genotoxicity - A Predict. Risk to Our Actual World, InTech; 2018; DOI:10.5772/intechopen.71923.

3. Sarmento B, Andrade F, Da Silva SB, Rodrigues F, Das Neves J, Ferreira D. Cell-based in vitro models for predicting drug permeability. Expert Opin Drug Metab Toxicol. 2012;8:607-21; DOI:10.1517/17425255.20 12.673586 .

4. Langhans SA. Three-dimensional in vitro cell culture models in drug discovery and drug repositioning. Front Pharmacol. 2018;9; DOI:10.3389/ fphar.2018.00006

5. Eisenbrand G, Pool-Zobel B, Baker V, Balls M, Blaauboer BJ, Boobis A, Carere A, Kevekordes S, Lhuguenot JC, Pieters R, Kleiner J. Methods of in vitro toxicology. Food Chem Toxicol. 2002;40:193-236; DOI:10.1016/ S0278-6915(01)00118-1.

6. Strober W. Trypan Blue Exclusion Test of Cell Viability. Curr Protoc Immunol. 2015;111:1-3; DOI:10.1002/0471142735.ima03bs111.

7. Kim SI, Kim HJ, Lee H-J, Lee K, Hong D, Lim H, Cho K, Jung N, Yi YW. Application of a non-hazardous vital dye for cell counting with automated cell counters. Anal Biochem. 2016;492:8-12; DOI:10.1016/j. ab.2015.09.010.

8. Bácskay I, Nemes D, Fenyvesi F, Váradi J, Vasvári G, Fehér P, Vecsernyés M, Ujhelyi Z. Role of Cytotoxicity Experiments in Pharmaceutical Development. Cytotoxicity, InTech; 2018; DOI:10.5772/intechopen.72539.

9. Fotakis G, Timbrell JA. In vitro cytotoxicity assays: Comparison of LDH, neutral red, MTT and protein assay in hepatoma cell lines following 
exposure to cadmium chloride. Toxicol Lett. 2006;160:171-7; DO I:10.1016/j.toxlet.2005.07.001.

10. Czekanska EM. Assessment of cell proliferation with resazurin -based fluorescent dye. Methods Mol Biol. 2011;740:27-32; DOI:10.1007/978-1-61779-108-6_5.

11. Kim HJ, Jang S. Optimization of a resazurin-based microplate assay for large-scale compound screenings against Klebsiella pneumoniae. 3 Biotech. 2018;8; DOI:10.1007/s13205-017-1034-9.

12. Lage OM, Ramos MC, Calisto R, Almeida E, Vasconcelos V, Vicente F. Current screening methodologies in drug discovery for selected human diseases. Mar Drugs. 2018;16; DOI:10.3390/md16080279.

13. Munshi A, Hobbs M, Meyn RE. Clonogenic cell survival assay. Methods Mol Med. 2005;110:21-8; DOI:10.1385/1-59259-869-2:021.

14. Franken NAP, Rodermond HM, Stap J, Haveman J, van Bree C. Clonogenic assay of cells in vitro. Nat Protoc. 2006;1:2315-9; DOI:10.1038/ nprot.2006.339.

15. Madhavan H. Simple Laboratory methods to measure cell proliferation using DNA synthesis property. J Stem Cells Regen Med. 2007;3:12-4.

16. Lyons AB, Blake SJ, Doherty K V. Flow cytometric analysis of cell division by dilution of CFSE related dyes. Curr Protoc Cytom. 2013; DOI:10.1002/0471142956.cy0911s64.

17. Romar GA, Kupper TS, Divito SJ. Research techniques made simple: Techniques to assess cell proliferation. J Invest Dermatol. 2016;136:e17; DOI:10.1016/j.jid.2015.11.020.

18. Jones JR, Lebar MD, Jinwal UK, Abisambra JF, Koren J, Blair L, O’Leary JC Davey Z, Trotter J, Johnson AG, Weeber E, Eckman CB, Baker BJ, Dickey CA. The diarylheptanoid (+)-aR,11S-myricanol and two flavones from bayberry (Myrica cerifera) destabilise the microtubule-associated protein tau. J Nat Prod. 2011;74:38-44; DOI:10.1021/np100572z.

19. Chakrabarti E, Smith J. Drug Library Screen to Identify Compounds that Decrease Secreted A\&\#946; from a Human Cell Line. Curr Alzheimer Res. 2005;2:255-9; DOI:10.2174/1567205053585738.

20. Liu XP, Qian X, Xie Y, Qi Y, Peng MF, Zhan BC, Lou ZQ. Betaine suppressed $A \beta$ generation by altering amyloid precursor protein processing. Neurol Sci. 2014;35:1009-13; DOI:10.1007/s10072-014-1630-y.

21. Chen WL, Qian Y, Meng WF, Pang JY, Lin YC, Guan YY, Chen SP, Liu J, Pei Z Wang GL. A novel marine compound xyloketal B protects against oxidised LDL-induced cell injury in vitro. Biochem Pharmacol. 2009;78:94150; DOI:10.1016/j.bcp.2009.05.029.

22. Barber SC, Higginbottom A, Mead RJ, Barber S, Shaw PJ. An in vitro screening cascade to identify neuroprotective antioxidants in ALS. Free Radic Biol Med. 2009;46:1127-38; DOI:10.1016/j. freeradbiomed.2009.01.019.

23. Wang S nan, Li Q Jing M hua, Alba E, Yang X hong, Sabaté R, Han Y fan, Pi R biao, Lan W jian, Yang X bo, Chen J kao. Natural Xanthones from Garcinia mangostana with Multifunctional Activities for the Therapy of Alzheimer's Disease. Neurochem Res. 2016;41:1806-17; DOI:10.1007/ s11064-016-1896-y.

24. Ock J, Suk K. Anti-inflammatory effects of synthetic compound KT14480 in lipopolysaccharide-stimulated microglia cells. J Pharm Pharmacol. 2010;62:279-85; DOI:10.1211/jpp.62.02.0017.

25. Cabrera-Pérez MÁ, Sanz MB, Sanjuan VM, González-Álvarez M, Álvarez IG. Importance and applications of cell-and tissue-based in vitro models for drug permeability screening in early stages of drug development. Concepts Model. Drug Permeability Stud. Cell Tissue based Vitr. Cult. Model., Elsevier Inc.; 2015, p. 3-29; DOI:10.1016/ B978-0-08-100094-6.00002-X.

26. Balimane $\mathrm{P}$ V., Han YH, Chong S. Current industrial practices of assessing permeability and P-glycoprotein interaction. AAPS J. 2006;8; DOI:10.1208/aapsj080101.

27. Martinez MN, Amidon GL. A mechanistic approach to understanding the factors affecting drug absorption: A review of fundamentals. J Clin Pharmacol. 2002;42:620-43; DOI:10.1177/00970002042006005.

28. Artursson P. Epithelial transport of drugs in cell culture. I: A model for studying the passive diffusion of drugs over intestinal absorbtive (Caco-2) cells. J Pharm Sci. 1990;79:476-82; DOI:10.1002/ jps.2600790604.

29. Volpe DA. Application of method suitability for drug permeability classification. AAPS J. 2010;12:670-8; DOI:10.1208/s12248-010-9227-8.

30. Mangas-Sanjuan V, González-Álvarez I, González-Álvarez M, Casabó VG Bermejo M. Modified Nonsink Equation for Permeability Estimation in Cell Monolayers: Comparison with Standard Methods. Mol Pharm. 2014;11:1403-14; DOI:10.1021/mp400555e.

31. Fearn RA, Hirst BH. Predicting oral drug absorption and hepatobiliary clearance: Human intestinal and hepatic in vitro cell models. Environ Toxicol Pharmacol. 2006;21:168-78; DOI:10.1016/j.etap.2005.06.002.

32. Sambuy Y, De Angelis I, Ranaldi G, Scarino ML, Stammati A, Zucco F. The Caco- 2 cell line as a model of the intestinal barrier: Influence of cell and culture-related factors on Caco-2 cell functional characteristics. Cell Biol Toxicol. 2005;21:1-26; DOI:10.1007/s10565-005-0085-6.

33. Gomes MJ, Mendes B, Martins S, Sarmento B. Cell-based in vitro model for studying blood-brain barrier (BBB) permeability. Concepts Model. Drug Permeability Stud. Cell Tissue based Vitr. Cult. Model., Elsevier Inc.; 2015, p. 169-88; DOI:10.1016/B978-0-08-100094-6.00011-0.

34. Sarmento B. Concepts and Models for Drug Permeability Studies: Cell and Tissue based In vitro Culture Models. Elsevier Inc.; 2015; DOI:10.1016/C2014-0-01137-9.

35. Sivandzade F, Cucullo L. In-vitro blood-brain barrier modeling: A review of modern and fast-advancing technologies. J Cereb Blood Flow Metab. 2018;38:1667-81; DOI:10.1177/0271678X18788769.

36. Edmondson R, Broglie JJ, Adcock AF, Yang L. Three-dimensional cell culture systems and their applications in drug discovery and cell-based biosensors. Assay Drug Dev Technol. 2014;12:207-18; DOI:10.1089/ adt.2014.573 